\title{
On the behavior of linear polarizers on highly focused radially polarized beams
}

David Maluenda, Artur Carnicer, Ignasi Juvells, Rosario Martínez-Herrero 


\title{
On the behavior of linear polarizers on highly focused radially polarized beams
}

\author{
David Maluenda $^{*}{ }^{a}$, Artur Carnicer ${ }^{b}$, Ignasi Juvells $^{b}$, Rosario Martínez-Herrero $^{a}$ \\ ${ }^{a}$ Universidad Complutense de Madrid, Facultad de Ciencias Físicas, Departamento de Óptica, \\ Ciudad Universitaria s/n, 28040 Madrid (Spain); \\ ${ }^{b}$ Universitat de Barcelona (UB), Facultat de Física, Departament de Física Aplicada, Martí i \\ Franquès 1, 08028 Barcelona (Spain)
}

\begin{abstract}
In this communication we analyze the light field distribution of a highly focused radially polarized beam when passes through a linear polarizer. The polarizer is modeled as a plane-parallel uniaxial absorbing medium with the optical axis parallel to the plate surfaces of the polarizer. Analytical results and numerical calculations are provided.
\end{abstract}

Keywords: Physical Optics, Polarizers, Highly focused beams

\section{INTRODUCTION}

In the last years, multiple applications of the polarization properties of electromagnetic field distributions generated in the focal region of an optical system with high numerical aperture (NA) have been described: electron acceleration, nonlinear optics, optical tweezers, etc. ${ }^{1-14}$ In this context, an important issue is to develop the theoretical framework to determine the behavior of a polarizer on a focused beam. ${ }^{15-17}$ We have recently published a study on how linear polarizers modify a tightly focused field. ${ }^{18}$ The polarizer was modeled as a plane-parallel uniaxial absorbing medium with the optical axis parallel to the plate surfaces of the polarizer. ${ }^{19-22}$ We found that the electric field component in the direction of propagation and the influence of the Fresnel coefficients modify the performance of the polarizer.

In the present paper, we analyze how radially polarized beams are modified when they passes through a linear polarizer. The article is organized as follows. Section 2 reviews basic concepts on propagation of electric fields in the focal area. In section 3, we describe a theoretical model for O-type polarizers and the projection vector equation for the angular spectrum of plane waves are also considered. In particular, we focus our attention on radially polarized beams and we introduced two parameters that are useful to provide more insight on the behavior of the beam after crossing the polarizer. Finally, the conclusions are presented in section 4 .

\section{ELECTROMAGNETIC FIELDS IN THE FOCAL AREA}

The Richards-Wolf equation provides The relationship between the paraxial illuminating beam $\mathbf{E}_{i}=\left(E_{i x}, E_{i y}, 0\right)$ and the focused field distribution $\mathbf{E}$ is ${ }^{23}$

$$
\mathbf{E}(\mathbf{r})=A \int_{0}^{\theta_{M}} \int_{0}^{2 \pi} \mathbf{E}_{\mathbf{0}} \exp (-i k \mathbf{r} \cdot \mathbf{s}) \sin \theta \mathrm{d} \theta \mathrm{d} \varphi .
$$

Here $\mathbf{r}=(r, \phi, z)$ denotes the polar coordinates at the focal area, $A$ is a constant value, $k$ is the wave-number, $\theta_{M}$ is the semi-aperture angle (related to the numerical aperture (NA) by means of NA $=\sin \theta_{M}$ ), $\theta$ and $\varphi$ are the

Further author information: (Send correspondence to D. Maluenda)

E-mail: d.maluenda@ucm.es

Third International Conference on Applications of Optics and Photonics, edited by Manuel F. M. Costa,

Proc. of SPIE Vol. 10453, 104530M - @ 2017 SPIE · CCC code: 0277-786X/17/\$18 · doi: 10.1117/12.2271772

Proc. of SPIE Vol. 10453 104530M-1 
coordinates at the Gaussian sphere of reference and the wave-front vector $\mathbf{s}$ reads $\mathbf{s}=(\sin \theta \cos \varphi, \sin \theta \sin \varphi, \cos \theta)$ [see Fig. 1 in $^{11}$ ], $\mathbf{E}_{0}$ is the so-called vectorial angular spectrum, namely

$$
\mathbf{E}_{0}=f_{1} \mathbf{e}_{1}+f_{2} \mathbf{e}_{2}=\sqrt{\cos \theta}\left(\left(\mathbf{E}_{i} \cdot \mathbf{e}_{1}\right) \mathbf{e}_{1}+\left(\mathbf{E}_{i} \cdot \mathbf{e}_{2}^{i}\right) \mathbf{e}_{2}\right)
$$

where $f_{1}$ and $f_{2}$ are related to the azimuthal and radial transverse components of the incident transverse field $\mathbf{E}_{i}$ respectively. Vectors $\mathbf{e}_{1}, \mathbf{e}_{2}$ and $\mathbf{e}_{2}^{i}$ are described by

$$
\begin{array}{r}
\mathbf{e}_{1}(\varphi)=(-\sin \varphi, \cos \varphi, 0) \\
\mathbf{e}_{2}^{i}(\varphi)=(\cos \varphi, \sin \varphi, 0) \\
\mathbf{e}_{2}(\varphi, \theta)=(\cos \theta \cos \varphi, \cos \theta \sin \varphi,-\sin \theta) .
\end{array}
$$

When the incident beam is radially polarized the field in the focal region reads ${ }^{1-3,24}$

$$
\mathbf{E}(\mathbf{r})=A \int_{0}^{\theta_{M}} \int_{0}^{2 \pi} f_{2}(\theta, \varphi) \mathbf{e}_{2}(\theta, \varphi) \exp (-i k \mathbf{r} \cdot \mathbf{s}) \sin \theta \mathrm{d} \theta \mathrm{d} \varphi
$$

where

$$
f_{2}(\theta, \varphi)=\sqrt{\cos \theta} \mathbf{E}_{i}(\theta, \varphi) \cdot \mathbf{e}_{2}^{i}(\theta, \varphi)
$$

\section{BEHAVIOR OF IDEAL POLARIZER ON RADIALLY HIGHLY FOCUSED FIELDS}

We describe a linear polarizer as a uniaxial anisotropic plane-parallel media of thickness $L$. The optical axis is assumed to be parallel to the plate surfaces and multiple internal reflections are ignored. ${ }^{21,22}$ If the ordinary and extraordinary refractive indexes of the material are very similar, the incident and transmitted beams are related by

$$
\mathbf{P}_{\beta}\left[\mathbf{E}_{0}\right]=\exp \left(-i k \hat{n}_{o} L\right)\left(\mathbf{E}_{0} \cdot \mathbf{q}_{\mathbf{o}}\right) \mathbf{p}_{\mathbf{o}}+\exp \left(-i k \hat{n}_{e} L\right)\left(\mathbf{E}_{0} \cdot \mathbf{q}_{e}\right) \mathbf{p}_{e}
$$

where $\hat{n}_{o}=n_{o}-i \kappa_{o}$ and $\hat{n}_{e}=n_{e}-i \kappa_{e}$ are the ordinary and extraordinary complex refractive indices; $\mathbf{P}_{\beta}[]$ the linear operator that mathematically describes an ideal linear polarizer whose optical axis ( $c$-axis) is described by $(\cos \beta, \sin \beta, 0)$. Thus, vectors $\mathbf{q}_{o}, \mathbf{p}_{o}, \mathbf{q}_{e}$ and, $\mathbf{p}_{e}$ are given by

$$
\begin{aligned}
& \mathbf{q}_{o}=t_{s} \cos \psi \mathbf{e}_{1}-t_{p} \sin \psi \mathbf{e}_{2} \\
& \mathbf{p}_{o}=t_{s}^{\prime} \cos \psi \mathbf{e}_{1}-t_{p}^{\prime} \sin \psi \mathbf{e}_{2} \\
& \mathbf{q}_{e}=t_{s} \sin \psi \mathbf{e}_{1}+t_{p} \cos \psi \mathbf{e}_{2} \\
& \mathbf{p}_{e}=t_{s}^{\prime} \sin \psi \mathbf{e}_{1}+t_{p}^{\prime} \cos \psi \mathbf{e}_{2}
\end{aligned}
$$

where $\cos \psi$ and $\sin \psi$ read

$$
\begin{aligned}
& \cos \psi=\frac{\cos \theta_{0} \cos (\varphi-\beta)}{\sqrt{1-\sin ^{2} \theta_{0} \cos ^{2}(\varphi-\beta)}} \\
& \sin \psi=-\frac{\sin (\varphi-\beta)}{\sqrt{1-\sin ^{2} \theta_{0} \cos ^{2}(\varphi-\beta)}} .
\end{aligned}
$$

The Fresnel transmission formulae for the first surface of the polarizing plate read

$$
\begin{aligned}
t_{s} & =\frac{2 \cos \theta}{\cos \theta+n_{o} \cos \theta_{0}} \\
t_{p} & =\frac{2 \cos \theta}{\cos \theta_{0}+n_{o} \cos \theta}
\end{aligned}
$$


where $\theta_{0}$ is the refraction angle, i.e. $\sin \theta=n_{o} \sin \theta_{0}$. The Fresnel coefficients for the second surface are

$$
\begin{aligned}
t_{s}^{\prime} & =\frac{2 n_{o} \cos \theta_{0}}{\cos \theta+n_{o} \cos \theta_{0}} \\
t_{p}^{\prime} & =\frac{2 n_{o} \cos \theta_{0}}{\cos \theta_{0}+n_{o} \cos \theta} .
\end{aligned}
$$

O-type polarizers transmit ordinary waves and attenuates extraordinary ones, i.e. $\kappa_{o} \simeq 0$ and $\kappa_{e}>0$ and thus, $\mathbf{P}_{\beta}\left[\mathbf{E}_{0}\right]=\left(\mathbf{E}_{0} \cdot \mathbf{q}_{\mathbf{o}}\right) \mathbf{p}_{\mathbf{o}}$ and the polarizer axis direction is $(-\sin \beta, \cos \beta, 0)$. Accordingly, the electric field of a focused beam after the polarizer $\mathbf{P}_{\beta}[\mathbf{E}(\mathbf{r})]$ is obtained by projecting each contributions of the angular spectrum $\mathbf{P}_{\beta}\left[\mathbf{E}_{0}\right]$. In this case, the Richards-Wolf equation Eq.(4) reads ${ }^{18}$

$$
\mathbf{P}_{\beta}[\mathbf{E}(\mathbf{r})]=A \int_{0}^{\theta_{M}} \int_{0}^{2 \pi}\left[\left(\mathbf{E}_{0} \cdot \mathbf{q}_{\mathbf{o}}\right) \mathbf{p}_{\mathbf{o}}\right] \exp (-i k \mathbf{r} \cdot \mathbf{s}) \sin \theta \mathrm{d} \theta \mathrm{d} \varphi .
$$

In particular, for incident radially polarized beam Eq. (11) reduces to

$$
\mathbf{P}_{\beta}[\mathbf{E}(\mathbf{r})]=A \int_{0}^{\theta_{M}} \int_{0}^{2 \pi} f_{2}(\theta, \varphi)\left[\left(\mathbf{e}_{2} \cdot \mathbf{q}_{\mathbf{o}}\right) \mathbf{p}_{\mathbf{o}}\right] \exp (-i k \mathbf{r} \cdot \mathbf{s}) \sin \theta \mathrm{d} \theta \mathrm{d} \varphi .
$$

From the previous equation it follows that the field after the polarizer is non uniform totally polarized and its features depend on the topological charge of the vector angular spectrum and the numerical aperture of the objective lens $\mathrm{NA}=\sin \theta_{M}$. Furthermore, Eq. (12) clearly shows that the field after the polarizer displays a non-negligible longitudinal component.

Figures 1(a) and 1(b) show the irradiance distributions of the focused electric field for a radially polarized beam, with rotational symmetry, before and after passing through a polarizer respectively. In what follows, the polarizer axis is set to the horizontal direction, i.e. $\beta=\pi / 2$. Moreover, the components images are normalized to the maximum value of the total irradiance $I_{T}$. The numerical aperture is set to NA=0.9. Figures display the irradiances of the $x$-component $I_{x}=\left|\mathbf{E}_{x}\right|^{2}, y$-component $I_{y}=\left|\mathbf{E}_{y}\right|^{2}, z$-component $I_{z}=\left|\mathbf{E}_{z}\right|^{2}$, the total field $I_{T}=I_{x}+I_{y}+I_{z}$ and the $3 \mathrm{D}$ polarization map. Since polarization axis is set in the horizontal direction thus, $I_{y} \approx 0$ as shown in Fig. 1(b). Because the irradiance of the longitudinal component is high, the total irradiance after the polarizer is quite different when compared with the $x$-component before the polarizer.

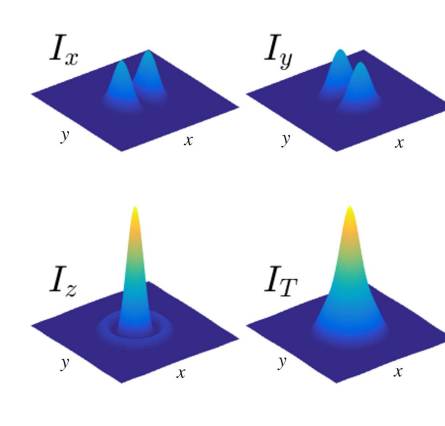

(a)

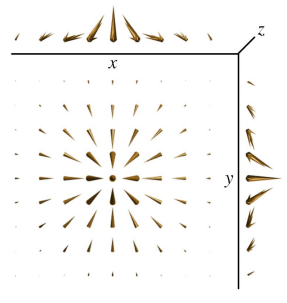

Pol. Map.

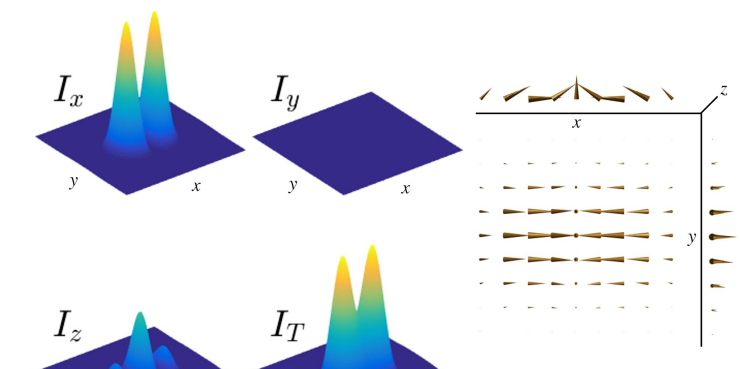

Pol. Map.

(b)

Figure 1: Radially polarized beam NA=0.9: (a) before and (b) after the polarizer. Both subfigures display the following distributions: $I_{x}, I_{y}, I_{z}, I_{T}$. In the polarization map: $x y$-plane, $x z$-profile and $y z$-profile are shown. 

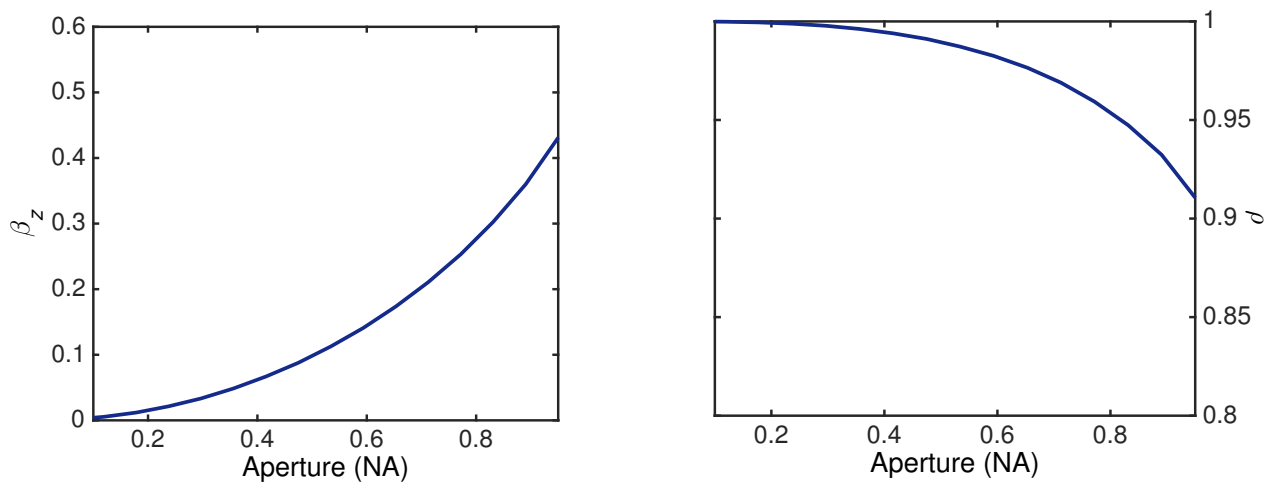

Figure 2: Behavior of the integrated irradiance parameters (left) $\beta_{z}$ and (right) $\rho$.

To better understand the behavior of radially polarized focused fields passing through a polarizer we introduce the following parameters: i) $\beta_{z}$ is the ratio of the integrated irradiances of the longitudinal component and the total field. Both distributions are evaluated after the polarizer, ii) $\rho$ is the correlation between the $x-$ component of the field before the polarizer and the total amplitude after the polarizer. This parameter ranges from $0 \leq \rho \leq 1$ in particular, if $\rho=1$ then both distributions are indistinguishable. Note that $\rho$ is closely related to the similarity factor introduced in ${ }^{25}$

$$
\begin{gathered}
\beta_{z}=\frac{\iint\left|\mathbf{P}_{\beta}[\mathbf{E}(\mathbf{r})]_{z}\right|^{2} \sin \phi d r d \phi}{\iint\left|\mathbf{P}_{\beta}[\mathbf{E}(\mathbf{r})]\right|^{2} \sin \phi d r d \phi} \\
\rho=\frac{\iint\left|\mathbf{P}_{\pi / 2}[\mathbf{E}(\mathbf{r})]\right|\left|E_{x}(\mathbf{r})\right| \sin \phi d r d \phi}{\sqrt{\iint\left|\mathbf{P}_{\pi / 2}[\mathbf{E}(\mathbf{r})]\right|^{2} \sin \phi d r d \phi} \sqrt{\iint\left|E_{x}(\mathbf{r})\right|^{2} \sin \phi d r d \phi}} .
\end{gathered}
$$

Figures 2 shows the behavior of these parameters as a function of NA. As expected, $\beta_{z}$ increases with NA and $\rho$ displays a monotonically decreasing behavior with NA. This behavior of $\rho$ is compatible with the results presented in Fig. 1. In particular, $I_{x}$ before and $I_{T}$ after the polarizer look quite different and consequently, an irradiance measurement of the focused beam after a polarizer can lead an unappropriated estimation of the $x$-component.

\section{CONCLUSION}

In this paper we discussed how a radially polarized focused beam is modified after passing through a linear polarizer. These devices are modeled as uniaxial anisotropic plane-parallel media with the optical axis parallel to the plate surfaces. It is worth to point out that the irradiance of the recorded beam after a polarizer differs from the projected component of the beam before passing the polarizer. In fact, differences can be observed when radially polarized beams are focused with high NA values. As a consequence, a measure of the irradiance of a focused beam after a polarizer can provide an inaccurate account of the projected component.

\section{Acknowledgments}

This research is funded by Ministerio de Economia y Competitividad (MINECO) project FIS2016-75147-C3-1-P.

\section{REFERENCES}

[1] Zhan, Q. and Leger, J., "Focus shaping using cylindrical vector beams," Opt. Express 10(7), 324-331 (2002).

[2] Davidson, N. and Bokor, N., "High-numerical-aperture focusing of radially polarized doughnut beams with a parabolic mirror and a flat diffractive lens," Opt. Lett. 29(12), 1318-1320 (2004).

[3] Kozawa, Y. and Sato, S., "Sharper focal spot formed by higher-order radially polarized laser beams," J. Opt. Soc. Am. A 24(6), 1793-1798 (2007). 
[4] Lindfors, K., Priimagi, A., Setälä, T., Shevchenko, A., Friberg, A. T., and Kaivola, M., "Local polarization of tightly focused unpolarized light," Nat. Photon. 1(4), 228-231 (2007).

[5] Zhan, Q., "Cylindrical vector beams: from mathematical concepts to applications," Adv. Opt. Photon. 1(1), $1-57$ (2009).

[6] Maluenda, D., Juvells, I., Martínez-Herrero, R., and Carnicer, A., "Reconfigurable beams with arbitrary polarization and shape distributions at a given plane," Opt. Express 21(5), 5432-5439 (2013).

[7] Serrels, K. A., Ramsay, E., Warburton, R. J., and Reid, D. T., "Nanoscale optical microscopy in the vectorial focusing regime," Nat. Photon. 2(5), 311-314 (2008).

[8] Wang, H., Shi, L., Lukyanchuk, B., Sheppard, C., and Chong, C. T., "Creation of a needle of longitudinally polarized light in vacuum using binary optics," Nat. Photon. 2(8), 501-505 (2008).

[9] Yang, L., Xie, X., Wang, S., and Zhou, J., "Minimized spot of annular radially polarized focusing beam," Opt. Letters 38(8), 1331-1333 (2013).

[10] Zha, Y., Wei, J., Wang, H., and Gan, F., "Creation of an ultra-long depth of focus super-resolution longitudinally polarized beam with a ternary optical element," J. Optics 15(7), 075703 (2013).

[11] Maluenda, D., Martínez-Herrero, R., Juvells, I., and Carnicer, A., "Synthesis of highly focused fields with circular polarization at any transverse plane," Opt. Express 22, 6859-6867 (Mar 2014).

[12] Martínez-Herrero, R., Juvells, I., and Carnicer, A., "Design of highly focused fields that remain unpolarized on axis," Opt. Letters 39(20), 6025-6028 (2014).

[13] Martínez-Herrero, R., Maluenda, D., Juvells, I., and Carnicer, A., "Experimental implementation of tightly focused beams with unpolarized transversal component at any plane," Opt. Express 22(26), 32419-32428 (2014).

[14] Carnicer, A., Juvells, I., Javidi, B., and Martínez-Herrero, R., "Optical encryption in the longitudinal domain of focused fields," Opt. Express 24(7), 6793-6801 (2016).

[15] Fainman, Y. and Shamir, J., "Polarization of nonplanar wave fronts," App. Opt. 23(18), 3188-3195 (1984).

[16] Aiello, A., Marquardt, C., and Leuchs, G., "Nonparaxial polarizers," Opt. Letters 34(20), 3160-3162 (2009).

[17] Korger, J., Kolb, T., Banzer, P., Aiello, A., Wittmann, C., Marquardt, C., and Leuchs, G., "The polarization properties of a tilted polarizer," Opt. express 21(22), 27032-27042 (2013).

[18] Martínez-Herrero, R., Maluenda, D., Juvells, I., and Carnicer, A., "Polarisers in the focal domain: Theoretical model and experimental validation.," Sci. Rep. 7, 42122 (2017).

[19] Yeh, P., "Electromagnetic propagation in birefringent layered media," J. Opt. Soc. Am. 69(5), 742-756 (1979).

[20] Yeh, P., "Optics of anisotropic layered media: a new 4×4 matrix algebra," Surf. Sci. 96(1), 41-53 (1980).

[21] Yeh, P., "Extended Jones matrix method," J. Opt. Soc. Am. 72(4), 507-513 (1982).

[22] Gu, C. and Yeh, P., "Extended Jones matrix method II," J. Opt. Soc. Am. A 10(5), 966-973 (1993).

[23] Wolf, E., "Electromagnetic diffraction in optical systems. I. An integral representation of the image field," P. Roy. Soc. London A Mat. 253(1274), 349-357 (1959).

[24] Martínez-Herrero, R. and Mejías, P., "Propagation of light fields with radial or azimuthal polarization distribution at a transverse plane," Optics express 16(12), 9021-9033 (2008).

[25] Martmez-Herrero, R., Juvells, I., and Carnicer, A., "On the physical realizability of highly focused electromagnetic field distributions," Opt. Lett. 38(12), 2065-2067 (2013). 